\title{
PhenX: Host: Biobehavioral measures for tobacco regulatory research
}

\author{
Gary A Giovino, ${ }^{1}$ Gary E Swan, ${ }^{2}$ Ben Blount, ${ }^{3}$ Stephanie O'Malley, ${ }^{4}$ Darigg C Brown, ${ }^{5}$ \\ Tabitha P Hendershot, ${ }^{5}$ on behalf of PhenX TRR Host: Biobehavioral Working Group
}

${ }^{1}$ Department of Health Behavior, University at Buffalo; SUNY, Buffalo, New York, USA

${ }^{2}$ Stanford Prevention Research Center, Stanford University School of Medicine, Stanford, California, USA

${ }^{3}$ Tobacco and Volatiles Branch, Division of Laboratory Sciences, CDC, Atlanta, Georgia, USA ${ }^{4}$ Psychiatry, Yale University, New Haven, Connecticut, USA

${ }^{5}$ RTI International, Research Triangle Park, North Carolina, USA

\section{Correspondence to}

Dr Gary A Giovino, Department of Health Behavior, University at Buffalo; SUNY, Buffalo, NY 14260, USA;

ggiovino@buffalo.edu

Received 28 January 2019 Revised 4 February 2019 Accepted 11 February 2019

\section{Check for updates}

(C) Author(s) (or their employer(s)) 2020. No commercial re-use. See rights and permissions. Published by BMJ.

To cite: Giovino $G A$, Swan GE, Blount B, et al. Tob Control

2020;29:s13-s19.

\section{ABSTRACT}

A working group (WG) of experts from diverse fields related to nicotine and tobacco addiction was constituted to identify constructs and measures for the PhenX (Phenotypes and eXposures) Tobacco Regulatory Research (TRR) Host: Biobehavioral Collection with potential relevance to users of both conventional and newer tobacco products. This paper describes the methods and results the WG used to identify, select, approve and place measures in the PhenX TRR Collection. The WG recognised 13 constructs of importance to guide their categorisation of measures already in the PhenX Toolkit ('complementary measures') and to identify novel or improved measures of special relevance to tobacco regulatory science. In addition to the 22 complementary measures of relevance to tobacco use already in the PhenX Toolkit, the WG identified and recommended nine additional Host: Biobehavioral measures characterising the use, exposure and health outcomes of tobacco products for application to TRR. Of these, five were self-administered or intervieweradministered measures: amount, type and frequency of recent tobacco use; flavor preference in e-cigarette users (adult and youth); pregnancy status and tobacco use; pregnancy status-mother and baby health and withdrawal from tobacco use. The remaining four measures were laboratory-based: cotinine in serum, expired carbon monoxide, 4-(methylnitrosamino)-1-(3pyridyl)-1-butanol in urine and cue reactivity. Although a number of validated tools are now available in the Host: Biobehavioral Collection, several gaps were identified, including a need to develop and test the identified measures in adolescent samples and to develop or identify measures of nicotine dependence, tolerance and withdrawal associated with newer non-combusted tobacco products.

\section{INTRODUCTION}

The US Food and Drug Administration's (FDA's) Center for Tobacco Products (CTP) and National Institutes of Health (NIH) initiated a project with the PhenX (Phenotypes and eXposures) development team to identify priority expert-recommended consensus measures for use in state, local and federal tobacco regulatory research (TRR). ${ }^{1}$ These collections of measures, meant to facilitate the combination or comparison of data and replication of findings, were added to the PhenX Toolkit as a freely available research resource. The FDA CTP, $\mathrm{NIH}$ and PhenX team convened a panel of senior scientific experts to provide guidance and oversight to the project. This TRR Panel (TRRP) used the Host, Agent, Vector and Environment (HAVE) model to provide a framework for conceptualising the various subdomains of the larger causal network encompassing current and future research to inform tobacco product regulation. ${ }^{2}$ In contrast to the decades of development and validation of hundreds of research tools to apply to cigarettes, comparatively few evidence-based tools are available to inform the regulation of all tobacco products, as required by the Family Smoking Prevention and Tobacco Control Act of 2009. 3-7

Delnevo and Bauer ${ }^{4}$ reviewed the state of the art (as of 2009) of the tobacco Host-related surveillance and evaluation measures contained in 10 major US national surveys, including the National Health Interview Survey (NHIS), the Behavioral Risk Factor Surveillance System, and the National Survey on Drug Use and Health (NSDUH). The inventory they presented (see table 1 of Delnevo and Bauer ${ }^{4}$ ) included 39 separate topic areas categorised into five domains: cigarette smoking behaviours, use of other tobacco products, youth issues, other and health behaviours/status/consequences. Delnevo and Bauer also discussed several methodological challenges and opportunities in Host-related surveillance (eg, rapid response surveillance, wireless substitution, declining response rates). Relevant to this paper, the authors recommended standardising both measures of tobacco products other than cigarettes and items administered in languages other than English. The authors called for increased coordination and dissemination among researchers through a network in which Host measures are catalogued and shared in the service of public health needs, such as tobacco monitoring and control. The current paper represents an important step towards the establishment of a collection of Host measures recommended to serve as a standard of comparison across studies.

\section{METHODS}

\section{Developing the scope of the Host: Biobehavioral Collection}

The PhenX TRR Host: Biobehavioral WG considered several potential measurement elements in an introductory call in June 2014 and an in-person meeting on 21 July 2014. Historically, research on nicotine and tobacco has emphasised Host characteristics that account for variation in the susceptibility to initiate and maintain the use of tobacco products (mostly combusted tobacco products). ${ }^{8-10}$ The WG considered the initial scope of the behavioural and biological factors that influence product use, exposure and potential or actual adverse health outcomes, which was identified 
Table 1 Complementary Host: Biobehavioral measures already in the PhenX Toolkit

\begin{tabular}{|c|c|}
\hline PhenX measure (PhenX id) & $\begin{array}{l}\text { Domain/ } \\
\text { collection name }\end{array}$ \\
\hline $\begin{array}{l}\text { Tobacco-Nicotine Dependence (031001) } \\
\text { Smoking Status (Adolescent 030603, Adult 030604) } \\
\text { Age of Initiation of Use (Adolescent 030701, Adult 030703) } \\
\text { Tobacco } 30 \text { day Quantity and Frequency (Adolescent 030803, } \\
\text { Adult 030804) } \\
\text { Tobacco-Age of Offset of Cigarette Use (Adolescent 030903; } \\
\text { Adult 030902) } \\
\text { Substances-Lifetime Abuse and Dependence (031401) }\end{array}$ & $\begin{array}{l}\text { Alcohol, tobacco } \\
\text { and other } \\
\text { substances } \\
\text { (Domain) }\end{array}$ \\
\hline Self-reported Craving (520306) & $\begin{array}{l}\text { SAA (Collection): } \\
\text { Substance-specific } \\
\text { intermediate } \\
\text { phenotypes }\end{array}$ \\
\hline $\begin{array}{l}\text { Patterns of Substance Use (Youth 510303, Adult 510304) } \\
\text { Family History of Substance Use Problems (Alcohol 510101, } \\
\text { Drugs 510102) } \\
\text { Substance Abuse and Dependence-Past Year-Tobacco (510403) }\end{array}$ & $\begin{array}{l}\text { SAA (Collection): } \\
\text { Assessment of } \\
\text { substance use and } \\
\text { substance use } \\
\text { disorders }\end{array}$ \\
\hline Behavioural Health Screener (560102) & $\begin{array}{l}\text { SAA (Collections): } \\
\text { Substance } \\
\text { use-related co- } \\
\text { morbidities and } \\
\text { health-related } \\
\text { outcome }\end{array}$ \\
\hline Tobacco (Non-Cigarette)-Product Use (081401) & $\begin{array}{l}\text { Oral health } \\
\text { (Domain) }\end{array}$ \\
\hline Smoking Quit Attempts (071001) & Cancer (Domain) \\
\hline Urine Assay for Tobacco Smoke Exposure (091701) & $\begin{array}{l}\text { Respiratory } \\
\text { (Domain) }\end{array}$ \\
\hline General Psychiatric Assessment (Child 120102, Adult 120101) & $\begin{array}{l}\text { Psychiatric } \\
\text { (Domain) }\end{array}$ \\
\hline $\begin{array}{l}\text { Physical, Social, and Mental Health Functioning (SF-36v2 }{ }^{\circledR} \text {, } \\
\text { 191201) }\end{array}$ & $\begin{array}{l}\text { Gastrointestinal } \\
\text { (Domain) }\end{array}$ \\
\hline
\end{tabular}

by the TRRP and found to be large and diverse (table 2). The breadth of the possible universe of measures required the recruitment of WG members with expertise in a wide range of topic areas. The Host: Biobehavioral WG members completed their reviews and recommendations of measures and protocols for the Toolkit between 20 June 2014 and 16 December 2014.

\section{Expertise of the Host: Biobehavioral WG}

The Host: Biobehavioral WG consisted of eight experts in this field of research who either held tenured positions as academic researchers at universities across the USA, were in clinical medical

\begin{tabular}{ll}
\hline Table 2 & Initial Host: Biobehavioral scope \\
\hline Behavioural factors & Biological factors \\
\hline Relapse & Flavour preference \\
\hline $\begin{array}{l}\text { Withdrawal } \\
\text { Co-occurring use of other tobacco products }\end{array}$ & $\begin{array}{l}\text { Biomarkers of exposure/harm } \\
\text { Nicotine metabolism }\end{array}$ \\
Cue reactivity/craving & $\begin{array}{l}\text { Health effects (short and } \\
\text { long term) }\end{array}$ \\
\hline Dependence & $\begin{array}{l}\text { Health status (pre-exposure } \\
\text { and post-exposure to tobacco } \\
\text { products and their by- } \\
\text { products) }\end{array}$ \\
\hline Topography of tobacco product use, including & Pregnancy status \\
patterns of use & Genetic factors \\
\hline
\end{tabular}

practice or were researchers at federal agencies. The WG was supported by four liaisons from the TRRP, one Special Consultant representing the Tobacco Centers of Regulatory Science (TCORS) and two federal agency experts who provided input on the federal government's tobacco regulatory priorities, how they relate to current biological and behavioural research portfolios and the measures that can inform regulatory activities. Each WG member had an established research portfolio and expertise in the descriptive, biological and behavioural risk factors for tobacco use and the impact of those factors on the tobacco regulatory environment. Two members were recruited as co-chairs of the WG to lead the consensus process and provide guidance for the selection of standardised measures. The members of the WG are listed in the Collaborators addendum to this manuscript.

The expertise of the WG members spanned several areas of inquiry, including health disparities, nicotine dependence, coping, cancer risk, epidemiology, survey research, health behaviour, the measurement of biomarkers and environmental exposures, the development of phenotypes and gene-environment interactions and psychopharmacology. This varied expertise helped to ensure adequate coverage of the most pressing areas for which standard measures and protocols were lacking. Some members had experience with special populations (eg, adolescents, young adults, substance abusers). The breadth and depth of the WG's expertise helped fulfil the aim of PhenX to expand the scope of the Toolkit, establish recommended measures in support of TRR and accelerate the integration of biobehavioural measures into new and ongoing TRR studies.

\section{Complementary measures already in the PhenX Toolkit}

The WG began the process of selecting measures by focusing on what was already in the Toolkit. This strategy provided insight into where the WG should focus their efforts to identify new measures that were not already addressed by the Toolkit. The measures listed in table 1 were already in the Toolkit when the WG was formed and were deemed to be complementary to the group's scope. Currently, these measures exist in nine different domains or collections on the PhenX website. They are accessible through the PhenX website and, along with the protocols described in the next section, comprise the full list of recommended Host: Biobehavioral measures.

As part of the WG's deliberation process, the measures proposed for inclusion in the PhenX Toolkit were disseminated to the scientific community for review and comment between 27 October and 12 November 2014. The outreach was sent to various email lists and listservs, including registered users of the Toolkit, members of professional societies, investigators funded to conduct TRR through the FDA-NIH partnership and experts who had participated in other PhenX WGs, panels or projects. WG and TRRP members and outreach email recipients were encouraged to forward the invitation to their colleagues to personalise the request for comments and encourage input from an audience targeted to the topic at hand. The comments received reflected broad and varied expertise and familiarity with the PhenX Toolkit. Prior to sending the emailed invitation, the primary authors of the measurement protocols suggested for inclusion in the Toolkit were contacted to seek permission and inform investigators of the potential for their protocol to be included in the PhenX Toolkit.

A total of 24 individuals provided comments on the proposed measures. Suggestions for revising the measures included the need to provide specific instructions on how to assess the concurrent use of multiple products and how to quantify the amount used 
of newer products, such as electronic cigarettes (e-cigarettes). In response, specific instructions were added to the measures of amount, type and frequency of recent tobacco product use. Other comments focused on the need for a methodology less cumbersome than the assay for cotinine in serum. However, protocols for assaying cotinine in urine and saliva already exist elsewhere in the Toolkit. A final question concerned the need for air quality monitoring to determine exposure to secondhand smoke (SHS). Again, such a measure was already available in the Toolkit within the Respiratory domain. The WG considered adding a measure of health status but found that health status was addressed by the Toolkit's Physical, Social, and Mental Health Functioning measure. The feedback collected helped the WG to refine the final set of measures for inclusion in the Toolkit.

\section{RESULTS}

The WG discussed the feedback obtained via the community outreach and finalised the measures to recommend for inclusion in the Toolkit. The recommendations were then considered and approved by both the TRRP and the PhenX Steering Committee. ${ }^{11}$ The final nine measures were released into the Toolkit on 20 February 2015 and are summarised in table 3. More complete details regarding the measures and protocols are provided in the subsequent text.

\section{Amount, type and frequency of recent cigarette use (PhenX id=720101)}

The Timeline Follow-Back (TLFB; available at https:// www. nova.edu/gsc/forms/timeline-followback-forms.html) protocol $^{1213}$ was recommended and approved to track the use of single or multiple tobacco products. Originally developed to measure alcohol use, ${ }^{12} 13$ this measure has been adapted to other substances, including cigarettes. ${ }^{14}{ }^{15}$ Using a calendar to guide the interviewer and participant, the TLFB traditionally tracks cigarette use for 30 days. It can be self-administered or administered by an interviewer. Data can be reliably collected via paper and pencil, telephone or computer. ${ }^{13}$ The TLFB has been validated with adolescent smokers. ${ }^{16}$ It involves asking participants to retrospectively estimate their cigarette use in the 14-30 days prior to the interview date. The TLFB makes substance-relevant adaptations in administration mode. For instance, for cigarettes and marijuana, individuals are asked to estimate the number of cigarettes or joints smoked per day, whereas for cocaine, respondents are asked if they used cocaine on a given day. ${ }^{14}$ Although the protocol was developed to assess cigarette use, the members of the WG agreed that the protocol can be adapted, depending on the investigator's needs, to assess the use of other tobacco products, including the number and frequency of cigars, pipes, hookah, e-cigarettes (including product type), smokeless tobacco, dissolvables, bidis and kreteks used. The co-occurring use of multiple products can also be assessed via this protocol. The average estimated time to complete the interviewer-administered protocol depends on the length of the period observed (eg, $14 \mathrm{vs}$ 30 days), the number of products quantified and the frequency of use of each product. A 30-day assessment of one frequently used product is estimated to take less than $15 \mathrm{~min}$.

\section{Cotinine in serum (PhenX id=91703)}

The WG recommended the laboratory protocol for measuring serum cotinine used by the Centers for Disease Control and Prevention (CDC) for the National Health and Nutrition Examination Survey (NHANES; https://www.cdc.gov/nchs/ data/nhanes/nhanes_01_02/106_b_met_cotinine.pdf) and the
Table 3 The 10 Host: Biobehavioral measures recommended and approved for inclusion in the PhenX Toolkit

\begin{tabular}{|c|c|}
\hline Measure name (PhenX id) & Description of measurement protocol \\
\hline $\begin{array}{l}\text { Amount, Type, and } \\
\text { Frequency of Recent } \\
\text { Cigarette Use (720101) }\end{array}$ & $\begin{array}{l}\text { Self-based, interviewer-based or computer-based } \\
\text { protocol to assess the use of single or multiple tobacco } \\
\text { products over } 7 \text { days to } 2 \text { years prior to the interview }\end{array}$ \\
\hline $\begin{array}{l}\text { Biomarkers-Cotinine in } \\
\text { Serum (91703) }\end{array}$ & Laboratory-based protocol to measure serum cotinine \\
\hline $\begin{array}{l}\text { Biomarkers-Expired Carbon } \\
\text { Monoxide (720301) }\end{array}$ & $\begin{array}{l}\text { Interviewer-administered protocol to collect CO } \\
\text { samples }\end{array}$ \\
\hline $\begin{array}{l}\text { Biomarkers-NNAL in Urine } \\
\text { (720401) }\end{array}$ & $\begin{array}{l}\text { Laboratory-based protocol for the detection of } \\
\text { tobacco-specific alkaloids in urine }\end{array}$ \\
\hline $\begin{array}{l}\text { Cue Reaction to Tobacco } \\
\text { Stimuli (720501) }\end{array}$ & $\begin{array}{l}\text { Interviewer-based protocol to assess a participant's } \\
\text { reactions to tobacco-related stimuli }\end{array}$ \\
\hline $\begin{array}{l}\text { Flavor preference-e- } \\
\text { Cigarette Adult \& Youth } \\
\text { (720601 \& 720602) }\end{array}$ & $\begin{array}{l}\text { Adult-Self-administered questionnaire to determine } \\
\text { flavour preference when using e-cigarette products } \\
\text { Youth—Self-administered questionnaire to determine } \\
\text { flavour preference when using e-cigarettes }\end{array}$ \\
\hline $\begin{array}{l}\text { Pregnancy Status and } \\
\text { Tobacco Use (720801) }\end{array}$ & $\begin{array}{l}\text { Self-based or interviewer-based protocol to assess } \\
\text { pregnancy history and the use of tobacco and other } \\
\text { substances during pregnancy }\end{array}$ \\
\hline $\begin{array}{l}\text { Pregnancy Status-Mother } \\
\text { and Baby Health (720901) }\end{array}$ & $\begin{array}{l}\text { Self-administered protocol to assess problems during } \\
\text { pregnancy and after childbirth }\end{array}$ \\
\hline $\begin{array}{l}\text { Withdrawal from Tobacco } \\
\text { Use (721001) }\end{array}$ & $\begin{array}{l}\text { Self-based and observation-based protocol to assess } \\
\text { symptoms of withdrawal from tobacco use }\end{array}$ \\
\hline
\end{tabular}

Population Assessment of Tobacco and Health (PATH) Study; https://www.icpsr.umich.edu/icpsrweb/NAHDAP/studies/ 36498). Cotinine is a major metabolite of nicotine that can be used as a marker for active smoking and as an index for SHS exposure. Cotinine is generally preferred over nicotine for such assessments because of its substantially longer half-life. The estimated half-life of cotinine in serum is approximately $15-20$ hours, whereas that of nicotine is only $0.5-3$ hours. Cotinine can be measured in serum, urine or saliva, and the Toolkit includes protocols for measuring cotinine in urine (091701) and saliva (91702). The half-lives of cotinine in all three fluids are similar. ${ }^{17}$ Blood plasma or serum has been used as the fluid of choice for studies requiring a quantitative assessment of nicotine exposure. For that reason, serum was chosen as the matrix for the NHANES cotinine analyses $(\mathrm{ng} / \mathrm{mL})$. This protocol has been used to measure serum cotinine in NHANES since $1988^{17} 18$ and provides accurate (inaccuracy typically $<4 \%$ ) and precise (coefficient of variation typically $<6 \%$ ) data for nicotine exposure from all sources. ${ }^{17}$ Importantly, the method's accuracy has been demonstrated to not drift over time. Thus, the lower serum cotinine levels measured in the latest NHANES survey cycle relative to NHANES 1988-1994 are attributable to decreased SHS exposure, not drifting/inaccuracy of the analytical method. ${ }^{17}$ Additionally, this biomarker can be used to measure exposure to nicotine at all ages. Although the time to collect the serum sample is estimated to be less than 15 min, the laboratory assay requires specialised equipment (liquid chromatography and tandem mass spectrometry) and training (Clinical Laboratory Improvement Amendments [CLIA] certification and sample handling). In adolescent populations, saliva or urine samples would likely be preferred for the assessment of cotinine as collecting these samples is non-invasive, and the results obtained using these matrices are well correlated with serum cotinine. ${ }^{19}$ 


\section{Expired carbon monoxide (PhenX id=720301)}

The assessment of $\mathrm{CO}$ in expired breath samples (half-life: 4-6 hours) is widely used to evaluate past-24-hour combusted tobacco use ${ }^{20}$ and is validated in adults and adolescents. ${ }^{1921}$ The primary value of $\mathrm{CO}$ testing is to confirm the co-occurring use of one or more combusted tobacco products among people who are obtaining nicotine from one or more non-combusted sources. The protocol requires the use of a standard breath smokerlyzer into which the participant exhales. Subsequent CO concentrations are measured in units of $\mathrm{COppm} / \mathrm{COHb}$. The protocol takes less than 15 min to complete.

\section{NNAL in urine (PhenX id $=720401)$}

The WG recommended the detailed laboratory protocol for measuring urinary 4-(methylnitrosamino)-1-(3-pyridyl)-1-bu tanol (NNAL) used by the CDC (https://www.cdc.gov/nchs/ data/nhanes/nhanes_11_12/2011-12_Laboratory_Procedures_ Manual.pdf) to analyse urine specimens in NHANES and the PATH Study (https://www.icpsr.umich.edu/icpsrweb/NAHDAP/ studies/36498). NNAL is a major metabolite of 4-(methylnit rosamino)-1-(3-pyridyl)-1-butanone (NNK), the most potent pulmonary carcinogen of all tobacco-specific n-nitrosamines (TSNAs). ${ }^{22}$ TSNAs are known to be carcinogenic to many animal species and are classified as human carcinogens by the International Agency for Research on Cancer (IARC). ${ }^{23}$ These carcinogens are of special significance because they combine inherent potent pulmonary carcinogenic potential with high degrees of tobacco exposure specificity because they can be found only in tobacco and tobacco smoke. Measuring NNAL in urine $(\mathrm{ng} / \mathrm{L})$ is a reliable way to determine NNK exposure among smokers, nonsmokers exposed to SHS and people who use smokeless tobacco products (eg, chewing tobacco, snuff) ${ }^{24-26}$ but typically not those using only e-cigarettes or dissolvables. This protocol was used to measure urinary NNAL in NHANES from 2007 to 2014. ${ }^{27}$ This biomarker can be used to measure exposure to NNK in people of any age and provides accurate (bias typically $<4 \%$ ) and precise (coefficient of variation typically $<6 \%$ ) data on NNK exposure from all sources. ${ }^{28}$ Importantly, the method's accuracy has been demonstrated to not drift over time. Therefore, the lower NNAL levels measured in the latest NHANES survey cycle compared with NHANES 2007-2008 are attributable to decreased SHS exposure, not drifting/inaccuracy of the analytical method. Although the time to collect the urine sample is estimated to be less than $15 \mathrm{~min}$, the laboratory assay requires specialised equipment (liquid chromatography and tandem mass spectrometry) and training (CLIA certification and sample handling).

\section{Cue reactions to tobacco stimuli (PhenX id=720501)}

Determining cue reactivity (self-reported cravings and, optionally, heart rate and blood pressure responses) using a 'highburden' protocol was also recommended by the WG. The inclusion of the cue reactivity protocol was considered essential because of the ubiquity of smoking cues in the environment and the fact that reactions to these cues serve as triggers, leading to increased cigarette consumption. The protocol itself consists of a relaxation period followed by a neutral cues trial and culminating in a smoking cues trial (eg, lighting a cigarette, smelling the smoke). After each cue presentation, a craving score is recorded. The protocol has been validated for use in adults and adolescents. ${ }^{29-31}$ Successful execution of the protocol requires a smoking laboratory with good ventilation, a one-way mirror for observation, an intercom system to deliver instructions and a clock or timepiece. This interviewer-administered procedure takes more than $15 \mathrm{~min}$ to complete and is intended for use in current smokers only. The questionnaire to which the participant responds is known as the Brief Questionnaire for Smoking Urges (QSU Brief) and is also available in the PhenX Toolkit.

\section{Flavor preference}

The WG recommended a six-item adult (18 years and older) questionnaire to determine flavour preference when using e-cigarettes (PhenX id =7 20 601). A five-item youth (12-17 years of age) flavour preference protocol is also provided (PhenX $\mathrm{id}=7 \quad 20$ 602). This interviewer-administered questionnaire was originally developed for use by the PATH study's Wave 2 survey (https://www.reginfo.gov/public/do/PRAICList?ref_nbr= 201307-0925-001). The protocol asks whether the respondent began use with a flavoured product (ie, e-cigarette, e-cigarette cartridge or e-liquid) and, if so, which of seven different flavours, ${ }^{32}$ either singly or in combination, they used. Additional items query the current use of flavoured products. The WG further recommended that the PATH protocol for flavours in e-cigarettes could be modified to include flavours in other tobacco products, such as hookah, cigars, little cigars and dissolvables. The average time to complete this protocol is estimated to be less than $15 \mathrm{~min}$.

\section{Pregnancy status and tobacco use (PhenX $i d=720801$ )}

The protocol approved for use derives from the aforementioned PATH Study (see the Adult Extended Interview; https://www. reginfo.gov/public/do/PRAICList?ref_nbr=201307-0925-001). This eight-item interviewer-administered questionnaire assesses birth complications during any previous pregnancy. Cigarette use during the respondent's most recent pregnancy is queried, and if present, the number of cigarettes smoked per day is also queried. A unique feature of the item set is a question pertaining to the use of nine different non-cigarette tobacco products in addition to medicinal nicotine and other prescription smoking cessation medications during pregnancy. The protocol is estimated to take less than $15 \mathrm{~min}$ to complete.

Pregnancy status-mother and baby health (PhenX id=720901) The WG recommended a core set of six questions from the Pregnancy Risk Assessment Monitoring System (PRAMS; https:// www.cdc.gov/prams/questionnaire.htm) to assess for problems during and after pregnancy. The PRAMS core set of questions comprises reliable measures of pregnancy-related events and has been used widely for decades and is updated on a regular basis. Items include a checklist of 10 potential pregnancy complications (eg, vaginal bleeding, toxaemia) and several items concerning the baby's health. The WG also recommended three questions from the NHIS (1998 Sample Adult Family Prevention Supplement-Pregnancy and Smoking; https://www.cdc.gov/ nchs/nhis/tobacco/tobacco_questions.htm) to assess whether mothers stopped smoking cigarettes during their most recent pregnancies. The protocol can be either self-administered or interviewer-administered and is estimated to take less than 15 min to complete.

\section{Withdrawal from tobacco use (PhenX id=721001)}

The Minnesota Tobacco Withdrawal Scale-Revised ${ }^{33}$ provides a self-report protocol to assess the severity of 15 symptoms of withdrawal before and after quitting the use of tobacco products. The observer protocol assesses the severity of four symptoms and a confidence rating on the accuracy of the ratings. Participants provide severity ratings over the previous 24 hours, and 
observers provide ratings over the previous week. The participant and observer protocols have been validated for use with adults 18 years and older. ${ }^{33}$ The average estimated time to complete the protocol is $15 \mathrm{~min}$. The questionnaire is available online in 14 languages other than English (http://www.med.uvm.edu/behaviorandhealth/research/minnesota-tobacco-withdrawal-scale).

\section{DISCUSSION}

The deliberations of the PhenX TRR Host: Biobehavioral WG led to the addition of measures assessing variables at the intersection of behaviour and biology. This paper and work by the PhenX TRR Host: Social/Cognitive $\mathrm{WG}^{34}$ expand on Delnevo and Bauer's ${ }^{4}$ recommendation to develop a repository of Host-related measures. Users around the world have access to the Toolkit repository. This work also expands on the work presented in the 12th IARC Handbook of Cancer Prevention, titled 'Methods for Evaluating Tobacco Control Policies'. ${ }^{35}$ Section 3.1 describes key constructs in the natural history of tobacco use based on international surveys of adolescents (eg, Global Youth Tobacco Survey [GYTS], Health Behaviours of School-aged Children, European School Survey Project on Alcohol and Other Drugs) and adults (eg, Global Adult Tobacco Survey, International Tobacco Control Policy Evaluation Project, STEPwise Approach to Chronic Disease Risk Factor Surveillance, Smoking Toolkit Study), in addition to some surveys from the USA (eg, NHIS, NSDUH, Tobacco Use Supplement to the Current Population Survey [TUS-CPS], National Youth Tobacco Survey [NYTS]).

The worldwide increase in the use of electronic products that deliver nicotine in an aerosol ${ }^{36}$ and, more recently, the emergence of heated tobacco products ${ }^{37}$ and the use of non-combusted tobacco products (eg, snus ${ }^{38}$ ) indicate a strong need for validated measures of the use of products other than manufactured cigarettes. Studies investigating the use of these products will employ cotinine and/or CO, as relevant. International work with $\mathrm{CO}$ must consider that lactose intolerance can influence expired air $\mathrm{CO}^{39}$ and that cotinine levels might be influenced by genetic factors that seem to vary by race and ethnicity. For example, non-Hispanic blacks in the USA seem to metabolise nicotine and cotinine more slowly than other racial/ethnic groups in this country. ${ }^{40}$ Future research could address the issue of whether such differences are evident, for example, between blacks and whites living in Africa.

Studies may also be needed to assess the validity of measures of the use of chemicals other than nicotine, such as tetrahydrocannabinol, in electronic devices. Recent publications describe recommendations for measuring chemical exposures resulting from the use of e-cigarettes ${ }^{42} 43$ and water pipes. ${ }^{44}$

The TLFB is a method for assessing the use of multiple products, particularly during change processes, such as switching from the use of one nicotine-delivering product to another, and for multiple product users. The TLFB provides more detail than is available in the quantity-frequency measures generally used by large tobacco-use surveys. It is, however, more time consuming to administer than quantity-frequency measures. For some products, particularly e-cigarettes, the best metric for defining quantity of use has not been established, and frequency of use may be more readily determined. A recent review ${ }^{45}$ highlights the need to measure the use of multiple tobacco products in more detail, suggesting that detailed measures, such as the TLFB, are needed.

Validation studies of the use of the TLFB, measures of cue reactivity, measures of tobacco use in pregnancy and withdrawal symptoms following the discontinuance of use are needed for products other than cigarettes. Furthermore, tolerance to cigarettes can be assessed via the Cigarette Evaluation Scale. ${ }^{46}$ To the best of our knowledge, no studies have validated the assessment of tolerance with other tobacco products.

Studies of dependence among users of smokeless tobacco products have produced mixed results. ${ }^{478}$ Additionally, research is emerging on measures of dependence on e-cigarettes ${ }^{49-52}$ and water pipes ${ }^{53}$ among adults. A recent analysis of data from Wave 1 2013-2014 of the PATH study identified a set of measures to assess tobacco dependence with similar discriminative ability across seven groups of users of various tobacco products (ie, cigarettes only, e-cigarettes only, cigars only, hookah only, smokeless only, cigarettes+e-cigarettes and multiple products). ${ }^{54}$ Twenty-four items selected from four instruments (ie, the Hooked on Nicotine Checklist [HONC], ${ }^{55}$ Wisconsin Inventory of Smoking Dependence Motives [WISDM], ${ }^{56} \mathrm{Nico}-$ tine Dependence Syndrome Scale (NDSS) ${ }^{57}$ and Diagnostic and Statistical Manual $[D S M]^{58}$ ) were examined using the graded item response model and differential item functioning analyses. The final scale consisted of questions from WISDM (11 items), NDSS (4 items) and DSM (1 item). The tobacco dependence construct identified exhibited concurrent validity with frequency of product use. The authors called for additional validation studies, including longitudinal analyses of PATH data to examine predictive validity. ${ }^{54}$ A separate effort has adapted the Patient-Reported Outcomes Measurement Information System (PROMIS) measures of dependence on cigarettes ${ }^{59} 60$ to assess dependence on e-cigarettes (PROMIS-E) ${ }^{61}$ The PROMIS nicotine dependence item bank includes 22-item, 7-item and 4-item versions appropriate for use with daily and non-daily smokers. The comparable versions of the PROMIS-E have demonstrated strong psychometric properties, including a confirmable, internally consistent latent structure that was measurement invariant by sex, race, e-cigarette use status, e-liquid nicotine content and cigarette smoking status; concurrent validity with the Penn State E-cigarette Dependence Index; and test-criterion relationships with vaping frequency, e-liquid nicotine concentration and e-cigarette quit attempts. The three versions were highly correlated, suggesting that the short versions can be used when brevity is important. The consistency of PROMIS-E and the original versions of the PROMIS measures for assessing nicotine dependence on cigarettes is an asset for studies of dual users of cigarettes and e-cigarettes.

The Host: Biobehavioral WG also discussed other considerations. Researchers have been interested in the methodological innovation of showing pictures depicting each product that is being assessed in the questionnaire. This strategy has been used in national studies, such as NHANES. Interested researchers can request access to the Roswell Park Tobacco Product Image Library, which includes brand images of products and is updated continuously, from Dr. Maansi Bansal-Travers at the Roswell Park Comprehensive Cancer Center (Maansi.Travers@RoswellPark.org).

Another consideration was that few scales for use with adolescents are included in the Toolkit. Validation work is needed for such scales to be added, through either the validation of existing measures for use in adolescents (eg, the Minnesota Tobacco Withdrawal Scale-Revised) or the development of protocols adapted or designed for use with adolescents. One recent example of such work is the construct validation study of the measure of cigarette dependence in the GYTS. ${ }^{62}$ Data from six countries (one from each WHO region) and nationally representative samples of adolescents were analysed to assess associations of the GYTS measure of time to first cigarette or craving for a cigarette. 
Those respondents who smoked during the previous 30 days were asked if they had ever had a cigarette or felt like having a cigarette first thing in the morning. The odds of responding positively to that question increased in a dose-response manner with frequency of smoking (as measured by days smoked/month) and intensity of smoking (as measured by cigarettes/day).

The WG also considered research gaps that could be addressed using validated measures. For example, the availability and marketing of non-combusted tobacco products might lower consumers' perceptions of the absolute risk of harm posed by the use of each of the various combusted tobacco products. Measures that appropriately assess perceived absolute risk of combusted products are needed before additional modified risk products are put on the market and allowed to make claims. Similarly, measuring on a population level whether former smokers' perceived susceptibility to relapse changes when modified risk products are marketed will be important. The current measure of susceptibility to initiate smoking is included on multiple nationally representative surveys, including the NSDUH and NYTS. ${ }^{8}$ Researchers will also need validated measures of the relative satisfaction, harm and cost of various non-combusted products compared with combusted products. As the PhenX Toolkit recommends a targeted list of measures to provide a standard of comparison across studies, the WG prioritised protocols for inclusion in the Toolkit that are well established, validated, reliable and/or accepted by the research community to ensure that the protocols will remain relevant over time.

In conclusion, the PhenX TRR Host: Biobehavioral WG added several important measures to the PhenX Toolkit; recognised the need for the development and validation of measures of dependence, tolerance and withdrawal for newer non-combusted tobacco products and recommended the inclusion of prioritised

\section{What this paper adds}

- The biobehavioural characteristics of the tobacco product user (ie, amount, type and frequency of recent tobacco product use; flavor preferences; pregnancy status; history of withdrawal from tobacco product use; and reactivity to tobacco product cues) and selected biomarkers of exposure (ie, cotinine in serum, expired carbon monoxide and 4-(methylnitrosamino)-1-(3-pyridyl)-1-butanol in urine) are well known to influence the initiation, maintenance and/or consequences of the use of tobacco products and, thus, are highly relevant to tobacco regulatory research (TRR).

- The continuous proliferation of novel tobacco products as advertised and marketed across various platforms to individuals who vary in these biobehavioural characteristics introduces challenges to scientists seeking to support evidence-based regulatory decision-making because suitable measures may not be readily available or widely known.

- The PhenX TRR Host: Biobehavioral Working Group made consensus recommendations to investigators in the field concerning the best questionnaire-based, interview-based and laboratory-based methods to assess these individual characteristics and noted that additional measures will be needed for use in children.

- As tobacco regulatory science evolves, it will be important for investigators to consistently use valid and comprehensive measures of multiple tobacco product user characteristics and their influence on the initiation, maintenance and consequences of tobacco product use.
Host: Biobehavioral measures in surveillance studies monitoring the population effects of newly introduced products to address TRR gaps.

Acknowledgements We gratefully acknowledge the contributions of the anonymous members of the scientific community who commented on the measures proposed for inclusion. We also acknowledge the contribution of Deborah Maiese, RTI International, who led the recruitment of WG members and the orientation to the PhenX process, and the contributions of the RTI editorial team, including Michelle Bogus, Debbie Bond, Judy Cannada and Amy Morrow, for providing expert editorial review of the manuscript.

Collaborators The following are members of the PhenX TRR Host: Biobehavioral WG. Co-Chairs: Anne M Joseph, University of Minnesota and SO, Yale University; WG Members: BB, CDC, Suzanne M Colby, Brown University, GAG, University at Buffalo, Stephen Hecht, University of Minnesota and Peter Shields, The Ohio State University; Special Consultant: Bonnie Halpern-Felsher, Stanford University; TRRP Liaisons: GES, Stanford Prevention Research Center, Kevin P Conway, National Institute on Drug Abuse, Robin J Mermelstein, University of Illinois at Chicago and Gordon B Willis, National Cancer Institute; Federal Agency Liaison: Lisa Postow, National Heart, Lung, and Blood Institute; NIH Project Coordinator: Kay L Wanke, NIH Office of Disease Prevention and the PhenX team from RTI International, including Tabitha Hendershot, TRR RTI Project Lead, DCB, WG Supervisor and Lisa Kilpatrick, WG Manager.

Contributors GES, TPH and KLW led the conceptualisation of the manuscript and the coordination of the writing team. GAG and GES drafted the scientific content of the manuscript, and DCB and TPH drafted the Methods and Results sections of the manuscript. BB, SO and KLW reviewed the draft and provided substantive revisions. $S M C$ reviewed the draft and provided comments. The Co-Chairs and Members of the PhenX TRR Host: Biobehavioral WG identified and proposed preliminary measures and voted on final measures included in the PhenX TRR Host: Biobehavioral Specialty Collection. TRRP members KPC, GES, RJM and GBW ensured that the WG process maintained fidelity with overall project goals. Federal Agency Liaison Lynn C Hull, FDA CTP, ensured project consistency with agency goals and priorities. NIH Project Coordinator KLW proposed the PhenX TRR initiative and contributed to its execution and completion. The PhenX team coordinated and facilitated the WG process, including project oversight and leadership (TPH), supervisory management (DSN) and project management (RG). PhenX NIH Program Official Erin M Ramos, National Human Genome Research Institute (NHGRI), provided project guidance and funding coordination. Carol M Hamilton, RTI International, is the PhenX Principal Investigator and provided project guidance and supervision.

\section{Conceptualization}

Methodology

Funding Research reported in this publication was supported by grant numbers U41HG007050 and U41HG007050-01S1 from the NIH NHGRI and FDA CTP.

Disclaimer The content is solely the responsibility of the authors and does not necessarily represent the official views of the NIH, the CDC or the FDA.

Competing interests None declared.

Patient consent for publication Not required.

Provenance and peer review Not commissioned; externally peer reviewed.

\section{REFERENCES}

1 Swan GE, Hendershot TP, van Bemmel DM, et al. The PhenX toolkit: core measures for tobacco regulatory research. Tob Control 2019;28:s139-42.

2 Giovino GA, Biener L, Hartman AM, et al. Monitoring the tobacco use epidemic I. overview: optimizing measurement to facilitate change. Prev Med 2009;48:54-10.

3 U.S. Department of Health and Human Services, Food and Drug Administration. Family smoking prevention and tobacco control act. section 1. Public law 111-31, 22, 2009. Available: https://www.fda.gov/TobaccoProducts/Labeling/RulesRegulationsGuidance/ ucm262084.htm [Accessed 17 Oct 2018].

4 Delnevo CD, Bauer UE. Monitoring the tobacco use epidemic III: the host: data sources and methodological challenges. Prev Med 2009;48(1 Suppl):S16-23.

5 U S Department of Health and Human Services. The health benefits of smoking cessation: a report of the surgeon general. (DHHS publication No. (CDC) 90-8416. Rockville MD: U.S. department of health and Human services, public health service, centers for disease control, center for chronic disease prevention and health promotion, office on smoking and health, 1990.

6 United States Public Health Service. Office of the surgeon General., National center for chronic disease prevention and health promotion (U.S.). office on smoking and health. Preventing tobacco use among youth and young adults: a report of the surgeon General. Atlanta GA Washington DC: U S Dept of Health and Human Services, Public Health Service, Centers for Disease Control and Prevention, National Center for 
Chronic Disease Prevention and Health Promotion. For sale by the Supt. of Docs, U S. G P 0, 2012.

7 Garcia-Cazarin ML, Mandal RJ, Grana R, et al. Host-agent-vector-environment measures for electronic cigarette research used in NIH grants. Tob Control 2019;28:s181-7.

8 Pierce JP, Choi WS, Gilpin EA, et al. Validation of susceptibility as a predictor of which adolescents take up smoking in the United States. Health Psychol 1996;15:355-61.

9 Tyas SL, Pederson LL. Psychosocial factors related to adolescent smoking: a critical review of the literature. Tob Control 1998;7:409-20.

10 Hyland A, Li Q, Bauer J, et al. Predictors of cessation in a cohort of current and former smokers followed over 13 years. Nicotine Tob Res 2004;6:363-9.

11 Maiese DR, Hendershot TP, Strader LC, et al. PhenX - Establishing a consensus process to select common measures for collaborative research. RTI Press. 2013 October; (RTI Press Publication No. MR-0027-1310). Research Triangle Park, NC: RTI Press 2013.

12 Sobell LC, Sobell MB. Timeline followback: a technique for assessing self-reported alcohol consumption. In: Litten RZ, Allen J, eds. Measuring alcohol consumption: psychosocial and biological methods. Totowa NJ: Humana Press, 1992: 41-72.

13 Sobell LC, Brown J, Leo Gl, et al. The reliability of the alcohol Timeline Followback when administered by telephone and by computer. Drug Alcohol Depend 1996;42:49-54

14 Robinson SM, Sobell LC, Sobell MB, et al. Reliability of the Timeline Followback for cocaine, cannabis, and cigarette use. Psychol Addict Behav 2014;28:154-62.

15 Brown RA, Burgess ES, Sales SD, et al. Reliability and validity of a smoking timeline follow-back interview. Psychol Addict Behav 1998:12:101-12.

16 Lewis-Esquerre JM, Colby SM, Tevyaw Tracy O'Leary, et al. Validation of the timeline follow-back in the assessment of adolescent smoking. Drug Alcohol Depend 2005; 79:33-43.

17 Bernert JT, Turner WE, Pirkle JL, et al. Development and validation of sensitive method for determination of serum cotinine in smokers and nonsmokers by liquid chromatography/atmospheric Pressure ionization tandem mass spectrometry. Clin Chem 1997;43:2281-91.

18 Pirkle JL, Flegal KM, Bernert JT, et al. Exposure of the US population to environmental tobacco smoke: the third National Health and Nutrition Examination Survey, 1988 to 1991. JAMA 1996;275:1233-40.

19 Jarvis MJ, Tunstall-Pedoe $\mathrm{H}$, Feyerabend $\mathrm{C}$, et al. Comparison of tests used to distinguish smokers from nonsmokers. Am J Public Health 1987;77:1435-8.

20 Sandberg A, Sköld CM, Grunewald J, et al. Assessing recent smoking status by measuring exhaled carbon monoxide levels. PLoS One 2011;6:e28864.

21 Cummings SR, Richard RJ. Optimum cutoff points for biochemical validation of smoking status. Am J Public Health 1988;78:574-5.

22 Hecht SS, Carmella SG, Murphy SE, et al. A tobacco-specific lung carcinogen in the urine of men exposed to cigarette smoke. N Engl J Med 1993;329:1543-6.

23 International Agency for Research on Cancer. IARC monographs on the evaluation of carcinogenic risks to humans. smokeless tobacco and tobacco-specific nitrosamines. Lyon France: IARC, 2007.

24 Hecht SS. Human urinary carcinogen metabolites: biomarkers for investigating tobacco and cancer. Carcinogenesis 2002;23:907-22.

25 Ashley DL, O'Connor RJ, Bernert JT, et al. Effect of differing levels of tobacco-specific nitrosamines in cigarette smoke on the levels of biomarkers in smokers. Cancer Epidemiol Biomarkers Prev 2010:19:1389-98.

26 Hatsukami DK, Benowitz NL, Rennard SI, et al. Biomarkers to assess the utility of potential reduced exposure tobacco products. Nicotine Tob Res 2006:8:169-91.

27 Xia Y, Bernert JT, Jain RB, et al. Tobacco-specific nitrosamine 4-(methylnitrosamino)1-(3-pyridyl)-1-butanol (NNAL) in smokers in the United States: NHANES 2007-2008. Biomarkers 2011:16:112-9.

28 Xia Y, McGuffey JE, Bhattacharyya S, et al. Analysis of the tobacco-specific nitrosamine 4-(methylnitrosamino)-1-(3-pyridyl)-1-butanol in urine by extraction on a molecularly imprinted polymer column and liquid chromatography/atmospheric pressure ionization tandem mass spectrometry. Anal Chem 2005;77:7639-45.

29 Bidwell LC, Leventhal AM, Tidey JW, et al. Effects of abstinence in adolescent tobacco smokers: withdrawal symptoms, urge, affect, and CUE reactivity. Nicotine Tob Res 2013:15:457-64.

30 Sayette MA, Hufford MR. Effects of cue exposure and deprivation on cognitive resources in smokers. J Abnorm Psychol 1994;103:812-8.

31 Carter BL, Tiffany ST. Meta-analysis of cue-reactivity in addiction research. Addiction 1999:94:327-40.

32 Grana RA, Ling PM. "Smoking revolution": a content analysis of electronic cigarette retail websites. Am J Prev Med 2014:46:395-403.

33 Hughes JR, Hatsukami D. Signs and symptoms of tobacco withdrawal. Arch Gen Psychiatry 1986:43:289-94

34 Piper ME, Brown DC, Hendershot TP, et al. PhenX: host: social/cognitive measures for tobacco regulatory research. Tob Control 2019;28:s143-50.

35 International Agency for Research on Cancer. Methods for evaluating tobacco control policies. Lyon France: IARC, 2008.
36 U S Department of Health and Human Services. E-cigarette use among youth and young adults. A report of the surgeon general. Atlanta GA: U S Department of Health and Human Services, Centers for Disease Control and Prevention, National Center for Chronic Disease Prevention and Health Promotion, Office on Smoking and Health, 2016.

37 Caputi TL. Industry watch: heat-not-burn tobacco products are about to reach their boiling point. Tob Control 2016;26:609-10.

38 Henningfield JE, Fagerstrom KO. Swedish match company, Swedish snus and public health: a harm reduction experiment in progress? Tob Control 2001;10:253-7.

39 McNeill AD, Owen LA, Belcher M, et al. Abstinence from smoking and expired-air carbon monoxide levels: Lactose intolerance as a possible source of error. Am J Public Health 1990;80:1114-5.

40 Pérez-Stable EJ, Herrera B, Jacob P, et al. Nicotine metabolism and intake in black and white smokers. JAMA 1998;280:152-6.

41 Benowitz NL, Bernert JT, Caraballo RS, et al. Optimal serum cotinine levels for distinguishing cigarette smokers and nonsmokers within different racial/ ethnic groups in the United States between 1999 and 2004. Am J Epidemiol 2009;169:236-48.

42 Pearson JL, Hitchman SC, Brose LS, et al. Recommended core items to assess ecigarette use in population-based surveys. Tob Control 2018;27:341-6.

43 Weaver SR, Kim H, Glasser AM, et al. Establishing consensus on survey measures for electronic nicotine and non-nicotine delivery system use: current challenges and considerations for researchers. Addict Behav 2018:79:203-12.

44 Maziak W, Ben Taleb Z, Jawad M, et al. Consensus statement on assessment of waterpipe smoking in epidemiological studies. Tob Control 2017;26:338-43.

45 Farrelly MC, Chaloupka FJ, Berg CJ, et al. Taking stock of tobacco control program and policy science and impact in the United States. J Addict Behav Ther 2017;1.

46 Westman EC, Levin ED, Rose JE. Smoking while wearing the nicotine patch: is smoking satisfying or harmful? Clin Res 1992; 40 .

47 Mushtaq N, Beebe LA. A review of the validity and reliability of smokeless tobacco dependence measures. Addict Behav 2012;37:361-6.

48 Strong DR, Messer K, Hartman SJ, et al. Measurement of multiple nicotine dependence domains among cigarette, non-cigarette and poly-tobacco users: insights from item response theory. Drug Alcohol Depend 2015;152:185-93.

49 Etter J-F, Eissenberg T. Dependence levels in users of electronic cigarettes, nicotine gums and tobacco cigarettes. Drug Alcohol Depend 2015;147:68-75.

50 Foulds J, Veldheer S, Yingst J, et al. Development of a questionnaire for assessing dependence on electronic cigarettes among a large sample of ex-smoking e-cigarette users. Nicotine Tob Res 2015;17:186-92.

51 Liu G, Wasserman E, Kong L, et al. A comparison of nicotine dependence among exclusive e-cigarette and cigarette users in the path study. Prev Med 2017:104:86-91.

52 Rostron BL, Schroeder MJ, Ambrose BK. Dependence symptoms and cessation intentions among US adult daily cigarette, cigar, and e-cigarette users, 2012-2013. BMC Public Health 2016;16.

53 Maziak W, Eissenberg T, Ward KD. Patterns of waterpipe use and dependence: implications for intervention development. Pharmacol Biochem Behav 2005:80:173-9.

54 Strong DR, Pearson J, Ehlke S, et al. Indicators of dependence for different types of tobacco product users: descriptive findings from wave 1 (2013-2014) of the population assessment of tobacco and Health (path) study. Drug Alcohol Depend 2017:178:257-66

55 DiFranza JR, Savageau JA, Fletcher K, et al. Measuring the loss of autonomy over nicotine use in adolescents: the DANDY (development and assessment of nicotine dependence in youths) study. Arch Pediatr Adolesc Med 2002;156:397-403.

56 Piper ME, Piasecki TM, Federman EB, et al. A multiple motives approach to tobacco dependence: the Wisconsin inventory of smoking dependence motives (WISDM-68). J Consult Clin Psychol 2004;72:139-54.

57 Shiffman S, Waters A, Hickcox M. The nicotine dependence syndrome scale: a multidimensional measure of nicotine dependence. Nicotine Tob Res 2004;6:327-48.

58 American Psychiatric Association. Diagnostic and statistical manual of mental disorders. 5th edn. Arlington VA: American Psychiatric Publishing, 2013.

59 Edelen MO, Stucky BD, Hansen M, et al. The PROMIS smoking Initiative: initial validity evidence for six new smoking item banks. Nicotine Tob Res 2014;16:S250-60.

60 Shadel WG, Edelen MO, Tucker IS, et al. Development of the PROMIS nicotine dependence item banks. Nicotine Tob Res 2014;16:S190-201.

61 Morean M, Krishnan-Sarin S, Sussman S, et al. Psychometric evaluation of the patientreported outcomes measurement information system (PROMIS) nicotine dependence item bank for use with electronic cigarettes. Nicotine Tob Res 2018. doi:10.1093/ntr/ $\mathrm{nt} \times 271$

62 Lam E, Giovino GA, Shin M, et al. Relationship between frequency and intensity of cigarette smoking and TTFC/C among students of the GYTS in select countries, 20072009. J Sch Health 2014;84:549-58. 